\title{
Infrared resonance-enhanced multiphoton ionization spectroscopy of magnesium oxide clusters
}

\author{
D. van Heijnsbergen \\ FOM-Institute for Plasmaphysics Rijnhuizen, Edisonbaan 14, NL-3439 MN Nieuwegein, \\ The Netherlands and Department of Molecular and Laser Physics, University of Nijmegen, \\ Toernooiveld, NL-6525 ED Nijmegen, The Netherlands \\ G. von Helden \\ FOM-Institute for Plasmaphysics Rijnhuizen, Edisonbaan 14, NL-3439 MN Nieuwegein, The Netherlands
}

G. Meijer

FOM-Institute for Plasmaphysics Rijnhuizen, Edisonbaan 14, NL-3439 MN Nieuwegein, The Netherlands and Department of Molecular and Laser Physics, University of Nijmegen, Toernooiveld, NL-6525 ED Nijmegen, The Netherlands

M. A. Duncan a)

Department of Chemistry, University of Georgia, Athens, Georgia 30602

(Received 17 September 2001; accepted 14 November 2001)

\begin{abstract}
Neutral $(\mathrm{MgO})_{n}$ clusters are produced in a molecular beam by laser vaporization in a pulsed-nozzle cluster source. These clusters are ionized via multiphoton absorption from either an ultraviolet excimer laser or a far-infrared free electron laser. While ultraviolet ionization produces mass spectra consistent with previous measurements, infrared ionization produces higher molecular weight ions from the same nascent source distribution. Ultraviolet ionization occurs by direct electronic excitation/ionization, while infrared ionization occurs by vibrational excitation followed by thermionic electron emission. In both cases, prominent masses are observed corresponding to cubic nanocrystals with near equal $x: y: z$ dimensions. By tuning the IR wavelength while recording the mass-resolved ion yield, vibrational spectra are obtained revealing two resonances near 16 and 22 microns. Clusters up to 300 atoms in size are studied, and spectra exhibit a gradual variation with size, converging to positions near to, but not matching the bulk phonon frequencies. Structural implications of these vibrational spectra are investigated. (c) 2002 American Institute of Physics.
\end{abstract}

[DOI: $10.1063 / 1.1432999]$

\section{INTRODUCTION}

Since the first gas phase metal clusters were produced, a goal of their study has been the measurement of structure and energetics as a function of size and the comparison of these properties to those of the corresponding solid material. Unfortunately, this goal has rarely been realized. Electronic structure as a function of cluster size has been probed through ionization potential measurements, ${ }^{1,2}$ photoelectron spectroscopy, ${ }^{3-5}$ and photodissociation spectroscopy on plasmon resonances. ${ }^{6,7}$ However, measurements of vibrational or geometric structure have not been possible. Vibrational spectroscopy has been limited by the inavailability of tunable lasers in the required far-infrared wavelength region. There has also been no technique sensitive and selective enough to measure vibrational spectra for specific clusters formed at low density in a distribution of sizes. However, we have recently demonstrated that infrared resonance-enhanced multiphoton ionization (IR-REMPI) spectroscopy with a tunable free-electron laser can provide size-specific vibrational spectra for fullerenes ${ }^{8}$ and for metal-containing clusters. ${ }^{9-12} \mathrm{We}$ are exploring the application of this new technique for vari-

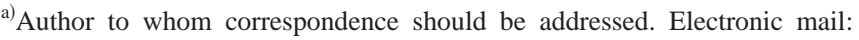
maduncan@arches.uga.edu
}

ous metal containing clusters. In this report, we describe new results for magnesium oxide clusters up to about 300 atoms in size.

Solid $\mathrm{MgO}$ is a well-studied material with numerous applications, ${ }^{13-19}$ especially as a support for metal nanoparticle catalysts. ${ }^{17}$ It also exhibits a variety of interesting surface chemistry. ${ }^{16,17}$ Its structure is face-centered cubic, even when colloidal size particles are produced, as in the familiar burning of magnesium ribbon. It is an insulator with a band gap of $7.8 \mathrm{eV} .{ }^{19} \mathrm{MgO}$ has a high melting point $\left(2826^{\circ} \mathrm{C}\right)$ and enthalpy of formation $\left(\Delta H_{f}^{0}(s)=-602 \mathrm{~kJ} / \mathrm{mol}\right)$, consistent with strong ionic bonding. ${ }^{19}$ Bulk and surface phonon vibrational spectra are well characterized via infrared reflectance ${ }^{15}$ and high resolution electron energy loss spectroscopy (HREELS) measurements on the solid surface, ${ }^{13,14}$ as well as IR absorption measurements on small particles. ${ }^{18}$

$\mathrm{MgO}$ molecules and clusters have been studied previously in the gas phase $\mathrm{e}^{20-26}$ and with $a b$ initio theory. ${ }^{27-33}$ The electronic spectrum of $\mathrm{MgO}$ is well known. ${ }^{21}$ Its dissociation energy is somewhat uncertain, but it lies in the $3.5-4.5 \mathrm{eV}$ range, ${ }^{20,21}$ while the ionization energy is $8.007 \mathrm{eV}^{22}$ Mass spectra of $(\mathrm{MgO})_{n}$ clusters have been studied, ${ }^{23,24}$ and photoelectron spectroscopy has been measured for small anion clusters. ${ }^{25,26}$ In the small size range, mass spectra exhibited 
prominent abundances at clusters with the stoichiometry of $(\mathrm{MgO})_{3 n}$. The $n=1$ cluster was calculated to have a sixmembered ring structure, and larger species were suggested to have stacked-ring structures denoted as nanotubes. ${ }^{23,24,33}$ In larger clusters, mass spectra suggest cubic nanocrystalline structures ${ }^{24}$ but no direct data has been obtained for either of these structural motifs. Although there are no previous studies of cluster bond energies, the strong bonding in the diatomic and in the solid suggests that $\mathrm{MgO}$ clusters will also have significant stability, making them good candidates for IR-REMPI measurements. MgO clusters have been proposed as carriers of IR bands observed for dust clouds near oxygen-rich stars. ${ }^{34-37}$ There is therefore considerable interest in their vibrational spectroscopy.

In the present work, vibrational spectra for $\mathrm{MgO}$ clusters are measured with the new IR-REMPI technique. ${ }^{8}$ This method is based on the ability of certain strongly bound clusters to exhibit "thermionic emission" of electrons following multiphoton laser excitation. In strongly bound clusters, ionization can be competitive with other photoinduced processes such as dissociation. Elimination of atoms by photodissociation often requires that multiple bonds be broken, and in general the energy required for evaporation of neutral fragments increases with cluster size. Ionization, on the other hand, becomes easier in larger clusters because IPs usually decrease with cluster size. Thermionic emission at fixed laser wavelengths has been observed for metal clusters, ${ }^{38,39}$ fullerenes, ${ }^{40,41}$ and metal carbide clusters. ${ }^{38,42,43}$ IR-REMPI is based on the observation that thermionic emission is enhanced when the laser is resonant with vibrational frequencies, and it requires a tunable, high fluence laser in the infrared and far-IR wavelength regions. IR-REMPI was first described as a spectroscopic tool for gas phase clusters in the study of $\mathrm{C}_{60} \cdot{ }^{8}$ In later work, we have employed this method to study transition metal carbide and oxide clusters produced in pulsed molecular beams by laser vaporization. ${ }^{9-12}$ In the present report, we extend this method to study the main group metal oxide clusters of $\mathrm{MgO}$. For the first time, clusters up to 300 atoms are studied to follow the variation of vibrational spectra with cluster size.

\section{EXPERIMENT}

Magnesium oxide clusters are produced in a molecular beam by laser vaporization in a pulsed nozzle source. The cluster source and molecular beam machine for these experiments have been described previously. ${ }^{9-12}$ A rotating Mg rod is vaporized with the focused output of a Nd:YAG laser (532 $\mathrm{nm})$ in an expansion of argon seeded with 1-5\% oxygen. The source employs a short-pulse gas valve (R. Jordan Co.) and either straight channel sections or a so-called waiting room to enclose the flowing plasma generated by the laser for cluster growth. The cluster beam passes through a skimmer as it enters a differentially pumped detection chamber, where its contents are measured with a reflectron time-offlight mass spectrometer (R. Jordan Co.). Cluster ions in the molecular beam are removed by deflection plates located upstream from the mass spectrometer. Neutral clusters are

\section{$(\mathrm{MgO})_{\mathrm{n}}$ Clusters}

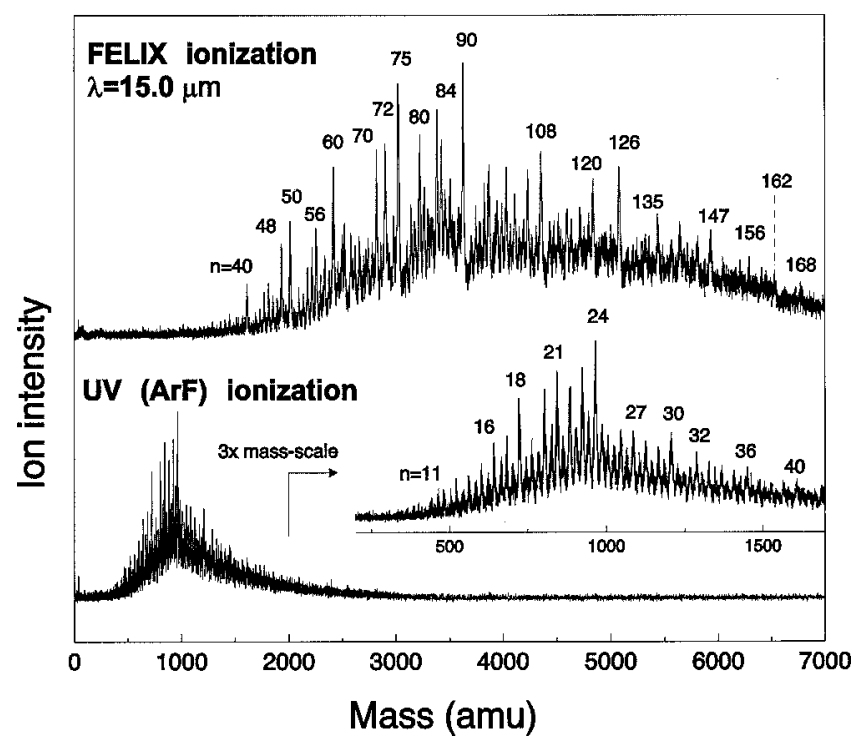

FIG. 1. A comparison of the mass spectra obtained with infrared multiphoton ionization with the free electron laser and $\mathrm{ArF}$ excimer laser multiphoton ionization. The prominent masses observed correspond to cubic nanocrystal structures.

sampled with multiphoton ionization at $\mathrm{KrF}(248 \mathrm{~nm})$ or $\mathrm{ArF}$ $(193 \mathrm{~nm})$ excimer laser wavelengths or with a tunable farinfrared free electron laser.

Infrared multiphoton ionization is accomplished with the free electron laser for infrared experiments (FELIX) ${ }^{44}$ The laser output comes in macropulses of $5 \mu$ s width at a repetition rate of $5 \mathrm{~Hz}$. Each macropulse contains a series of $0.5-5$ ps micropulses at a repetition rate of $1 \mathrm{GHz}$. A macropulse contains up to $100 \mathrm{~mJ}$ of energy and the bandwidth is transform limited. The possible tuning range is $40-2000 \mathrm{~cm}^{-1}$, although only the region from $400-1000 \mathrm{~cm}^{-1}$ is used in the present experiments. The IR beam is focused on the molecular beam with curved mirrors and fires at a time to intersect it in the time-of-flight source. A variable time delay after the IR laser pulse (typically $1-5 \mu \mathrm{s}$ ) is employed to allow thermal heating of the clusters to evolve into ionization, and then the TOF acceleration plates are pulsed to high voltage to extract cations from the molecular beam into the TOF mass spectrometer. The ion signal is recorded with a digital oscilloscope (Yokogawa DL 4200) interfaced to a laboratory PC. Full TOF mass spectra are recorded at each FELIX laser wavelength while the laser is scanned to obtain infrared resonance enhanced multiphoton ionization (IR-REMPI) spectra. Subsequent data analysis can extract the wavelength dependence of any cluster mass or the mass spectrum at any wavelength. Beam attenuators are used to study the power dependence of the IR-REMPI signal.

\section{RESULTS AND DISCUSSION}

Figure 1 shows the mass spectra measured for $(\mathrm{MgO})_{n}$ clusters using different ionization lasers. As shown, there are dramatically different mass spectra when we use the ultraviolet radiation from an $\operatorname{ArF}(193 \mathrm{~nm})$ excimer laser for ionization compared to thermionic ionization induced by the 
infrared radiation from FELIX. A mass spectrum similar to that obtained with $\mathrm{ArF}$ is also obtained using $\mathrm{KrF}$ ionization at $248 \mathrm{~nm}$ (data not shown). The UV ionization produces primarily cations in the small size range for $(\mathrm{MgO})_{n}$ species up to about $n=40$, whereas the IR-REMPI conditions produce ions up to beyond $n=160$. These mass spectra are significantly different, but the differences are understandable based on the ionization mechanism expected for the two different experiments. At the $\mathrm{ArF}$ or $\mathrm{KrF}$ laser wavelengths, the photon energy $(6.4$ or $5.0 \mathrm{eV})$ is not likely to be high enough to ionize these clusters with one photon. The IP of diatomic $\mathrm{MgO}$ is $8.0 \mathrm{eV}$, and the bulk work function can be estimated to be $6.5 \mathrm{eV}$ (vide infra). It is thus likely that the IPs of these clusters are between these values, and ionization with the excimer laser is therefore likely to be a multiphoton process. Consistent with this, UV ionization is inefficient except at high laser power. When the laser power is increased, absorption of photons beyond the minimum necessary for ionization leads to fragmentation, and stable cation species survive preferentially under these conditions. It has been shown in previous MPI studies of other metal clusters that the prominent masses are essentially the same as those produced when the clusters grow directly as cations. ${ }^{45}$ The more abundant clusters in the small size range can therefore be associated with stable cations. The infrared ionization process on the other hand is believed to occur as neutral clusters absorb energy gradually to heat the cluster. The vibrational energy must be coupled into the electronic degrees of freedom to achieve ionization. If fragmentation can occur, it will compete with ionization. Therefore, the abundant ions in this distribution are likely to represent the more stable neutral clusters in the nascent distribution. The IR ionization is also more efficient when clusters can accommodate energy effectively and for those clusters with lower ionization potentials. This explains in a general way why much larger clusters are detected with this method.

The infrared ionization process here has all the properties of thermionic electron emission. Most specifically, the ions require a time delay of a few microseconds after the initial excitation before they are formed and extracted. This is then the first report of thermionic emission for magnesium oxide clusters. In previous studies of fullerene and metal clusters that exhibit thermionic emission, it was generally believed that the bond energies for cluster dissociation were greater than cluster ionization potentials. ${ }^{8-12}$ It is therefore interesting to consider if this condition also applies here. Unfortunately, bond energies and ionization potentials have not been measured for $\mathrm{MgO}$ clusters. If we consider the $\mathrm{MgO}$ molecule itself, the ionization potential $(8.0 \mathrm{eV})$ is actually much greater than the dissociation energy (best estimate, $\left.D_{0}=4.3 \mathrm{eV}\right) .{ }^{20}$ However, these clusters are probably more like the bulk material than they are like the diatomic. The dissociation energy of the clusters can be related to the bulk sublimation energy for the process $\mathrm{MgO}(\mathrm{s}) \rightarrow \mathrm{MgO}(\mathrm{g})$. While not measured directly, this can be derived from the bulk and diatomic thermochemistry as $\Delta H_{s}$ $=+157.7 \mathrm{kcal} / \mathrm{mole}(6.84 \mathrm{eV}){ }^{20}$ The ionization energy of the clusters can be related to the bulk work function, which is also a number that is difficult to measure for metal oxides.

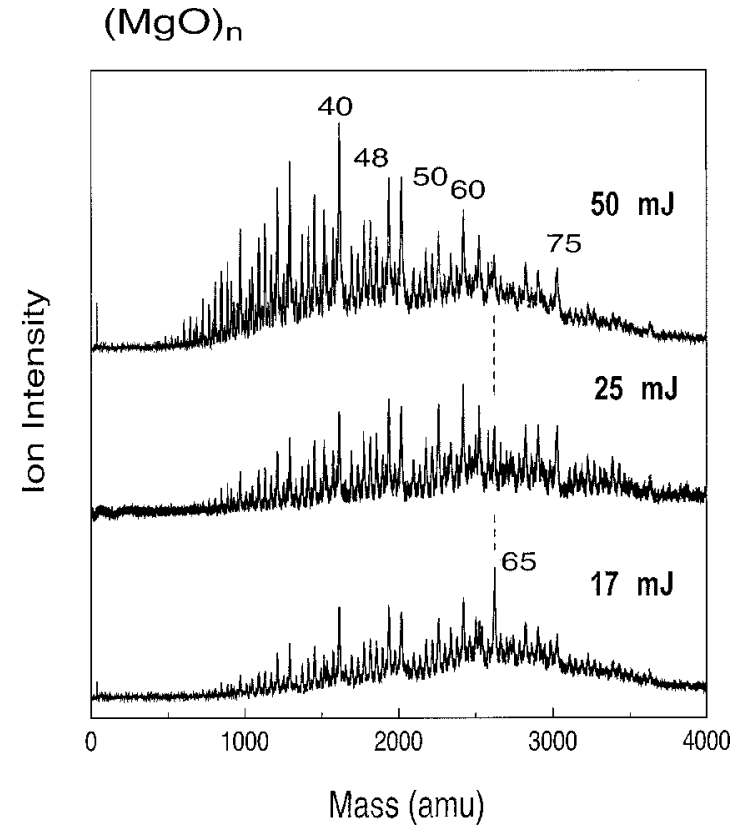

FIG. 2. The power dependence of the IR-REMPI mass distribution measured cumulatively over the wavelength range of 14.5 to 17.0 microns.

However, the band gap is well known $(7.8 \mathrm{eV}),{ }^{13}$ and the bulk electron affinity is estimated as less than $-1.3 \mathrm{eV},{ }^{13}$ from which the bulk work function is derived as $6.5 \mathrm{eV}$ or smaller. For the bulk then, the ionization energy appears to be slightly less than the fragmentation energy, and so these energetics should also be close for the clusters here. It is therefore not unreasonable that thermionic emission is observed. Indeed, secondary electron emission from $\mathrm{MgO}$ surfaces is known to be extremely efficient. ${ }^{13}$

In previous work, $(\mathrm{MgO})_{n}$ ions were produced via sputtering or by laser vaporization followed by ultraviolet laser multiphoton ionization. ${ }^{20-26}$ Both techniques produced cluster ions in the small size range with prominent masses for $(\mathrm{MgO})_{3 n}$. In combination with theory, the $(\mathrm{MgO})_{3}$ mass was attributed to a six-membered ring structure, and the $(\mathrm{MgO})_{3 n}$ species were assigned to stacked-ring or nanotube structures built from this same unit. A change in cluster structure was suggested to occur beyond the size of about $(\mathrm{MgO})_{30}$, where fcc nanocrystalline structures were inferred from the mass spectrum abundances. In the present data, the UV ionization mass spectra also show a somewhat enhanced abundance for the same $(\mathrm{MgO})_{3 n}$ mass peaks, as seen in the previous work. The IR-REMPI experiment also produces ions in the size range of $n=15-30$, where the proposed stacked-ring structures would be found. It is therefore possible to test for structural differences between small and large clusters using infrared spectroscopy.

In Fig. 2 we show a comparison of the IR-REMPI mass spectra measured at high, intermediate, and a low laser pulse energies $(50,25$, and $17 \mathrm{~mJ} /$ pulse), where the ion signal is summed over the range of 14.5 to 17.0 microns. These spectra have smaller clusters on average than that shown in Fig. 1 because of different source conditions. In each case the laser is focused, but the confocal waist varies significantly with wavelength, and so the absolute fluence for these spectra is 
TABLE I. The cubic structures giving rise to prominent $(\mathrm{MgO})_{n}$ stoichiometries.

\begin{tabular}{cccc}
\hline \hline$n$ & $x, y, z$ & $n$ & $x, y, z$ \\
\hline 15 & $2 \times 3 \times 5$ & 108 & $6 \times 6 \times 6,4 \times 6 \times 9$ \\
18 & $2 \times 3 \times 6,3 \times 3 \times 4$ & 126 & $6 \times 6 \times 7,4 \times 7 \times 9$ \\
21 & $2 \times 3 \times 7$ & 135 & $5 \times 6 \times 9$ \\
24 & $3 \times 4 \times 4,2 \times 3 \times 8$ & 147 & $6 \times 7 \times 7$ \\
30 & $3 \times 4 \times 5,2 \times 5 \times 6$ & 156 & $4 \times 6 \times 13,3 \times 8 \times 13$ \\
32 & $4 \times 4 \times 4$ & 162 & $6 \times 6 \times 9,4 \times 9 \times 9$ \\
40 & $4 \times 4 \times 5$ & & \\
50 & $4 \times 5 \times 5$ & & \\
60 & $4 \times 5 \times 6,3 \times 5 \times 8$ & & \\
65 & $2 \times 5 \times 13$ & & \\
72 & $4 \times 6 \times 6,3 \times 6 \times 8$ & & \\
90 & $5 \times 6 \times 6,4 \times 5 \times 9$ & & \\
\hline \hline
\end{tabular}

difficult to quantify. Nevertheless, there is clearly a shift in the cluster ion intensity across the distribution as the fluence is varied. One explanation for this is that fragmentation occurs in neutral clusters, followed by ionization of the fragments, or in ions by continued absorption after the ionization threshold. To limit the possible effects of fragmentation we measure our wavelength spectra at reduced laser power, although there is no change in any resonances with the laser fluence. Although the intensity distribution here shifts with fluence, the prominent mass peaks remain mostly the same in these spectra. The notable exception to this is the $n=65$ feature, which is hardly noticeable at high fluence and then becomes the largest peak in the spectrum at low fluence. On the other hand, it is also possible that the intensity shift across this distribution is not caused by fragmentation at all. If we examine the absolute signal levels, it is evident that the intensity at the smaller masses grows at higher laser power relative to the $n=65$ peak, but the higher masses have a nearly constant signal level. This is possible if the smaller clusters become ionized more efficiently as the fluence increases. The smaller clusters should have higher IPs and they would require more photons on average for ionization, and so it should be more difficult to ionize these species at low laser fluence. This is completely consistent with the thermionic emission mechanism.

The prominent masses that are labeled in Figs. 1 and 2 can all be rationalized in terms of fragments from the bulk fcc lattice. The most obvious way to accomplish this is by simple cubic structures with roughly equal $x, y, z$ dimensions, which corresponds to cutting the bulk crystal along the (100) planes. Table I shows some selected examples of $x, y, z$ values that could lead to such cubic structures. In many cases there is more than one way to arrive at a prominent stoichiometry. For example, the $n=60$ cluster could correspond to $4 \times 5 \times 6,3 \times 5 \times 8$, or $2 \times 5 \times 12$ lattices. However, the $4 \times 5 \times 6$ is considered to be the most likely because its symmetric dimensions minimize the surface free energy. Atoms along the edges of the structure have fewer bonds (4) than those in the face (5) or at interior sites (6) and therefore it is usually energetically favorable to have fewer of these. Another possible way to construct these clusters might be with layers corresponding to (111) planes of the solid. Symmetric layers occur for planes with 5, 13, 25, 41, 61,... atoms. However, to maintain the 1:1 stoichiometry, these layers should occur in pairs. It is then possible again to rationalize many of the prominent clusters with this scheme. For example, the $n$ $=60$ cluster could also be explained with four layers of 5 and four of 25. However, atoms along the sides of these (111) planes also have only four bonds, and so this configuration is generally considered to be less stable than corresponding simple cubic (100) configurations. Indeed, the (100) is the normal surface found for bulk $\mathrm{MgO}$ crystals, while the polar (111) face is found to be metastable. ${ }^{13}$

These issues of different fcc lattice fragments are interesting in particular because of the $n=65$ cluster. This is the one cluster that exhibits an extreme fluence dependence, becoming the largest peak in the mass spectrum at low fluence. It has a total of 130 atoms, which can only be rationalized as cubic if we assume dimensions of $2 \times 5 \times 13$. This cluster would look like a thin sheet or channel, which seems unlikely to form. It is conceivable that this cluster represents a cubic structure with nearly equal $x, y, z$ dimensions, but with a defect site with a few missing or extra atoms. For example, the $n=65$ cluster is only slightly larger than the $4 \times 4 \times 8$ structure $(n=64)$, and it may represent a species just beyond this more likely structure. However, there is no peak for the ideal $n=64$ cluster, and it is then not clear why such an intermediate species would be prominent. On the other hand, the $n=65$ species can also be explained with the (111) growth scheme, where it corresponds to either 10 layers of 13 , or to four layers of 25 and six layers of 5. These (111) arrangements would have unfavorable bonding compared to most (100) cubic structures, but they are probably more stable than the $2 \times 5 \times 13$ species. Another strange cluster appears with weak abundance at $n=156$, which can only be explained as cubic if the dimensions are $4 \times 6 \times 13$ or $3 \times 8$ $\times 13$. Again, this can alternatively be explained with a (111) pattern, as the $n=156$ cluster can be constructed from two layers of 61 atoms, four layers of 41 atoms, and two layers of 13 atoms. Thus, although most of the masses have simple explanations, others require more complex schemes. These mass spectra alone do not provide clear insight into the cluster structures.

Figure 3 shows the wavelength dependence of the IRREMPI signal for $(\mathrm{MgO})_{n}$ clusters in the small size range where $n=15,21,27$, and 32. The smaller clusters here often have $n$ as a multiple of three, and these species were proposed previously to represent stacked-ring nanotube structures. The signal-to-noise ratio in these data is not great due to the low abundance and/or low ionization efficiency of the small clusters. Additionally, there is an unavoidable oscillation in the signal due to precession of the sample rod in its mount. This oscillation is also seen in the larger cluster spectra shown below, but it is less of a problem because of the larger signals there. Although the data are noisy, it can be seen that there is resonance enhancement in the ionization yield. Two broad bands are observed near 16 and 22 microns, although the 22 micron feature is less apparent for the smaller clusters. In data not shown, these same broad resonances are also seen for the $n=18,24$, and 30 clusters, which were also proposed to have nanotube structures.

Figure 4 shows similar wavelength IR-REMPI spectra 


\section{$(\mathrm{MgO})_{\mathrm{n}}$ IR-REMPI}

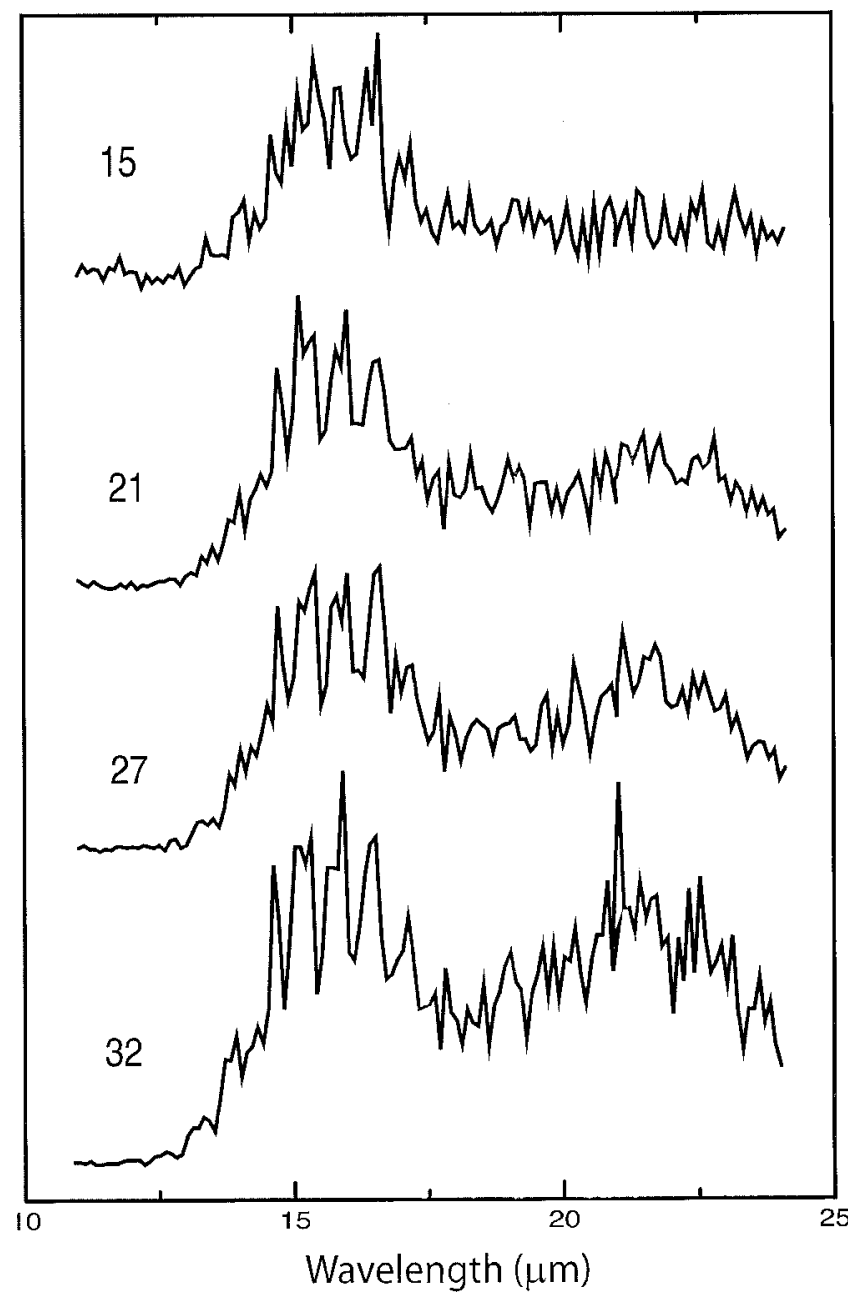

FIG. 3. The wavelength dependence of the IR-REMPI signal for the clusters in the small size range.

for larger $(\mathrm{MgO})_{n}$ clusters where $n=40,60,90,108,126$, and 168. It is remarkable that we are able to measure spectra with this method for clusters over the range of 30 atoms (at $n=15$ ) up to over 300 atoms. As indicated in the figure, the smaller clusters here (e.g., $n=40$ ) again have broad resonances near 16 and 22 microns just like the clusters in Fig. 3. However, the signal-to-noise ratio here is much improved, consistent with the larger mass peaks seen for these clusters in the mass spectra in Figs. 1 and 2. In larger clusters, the same broad resonances are measured, but the center of the bands shifts gradually in position. The shorter wavelength band appears to get broader in larger clusters, with its band center shifting very slightly toward longer wavelength. On going from the smallest cluster to the largest, the band center changes from about 15.5 to 16 microns. The longer wavelength feature is always broader and less intense than the shorter wavelength feature, and it shifts toward shorter wavelength. On going from the smallest cluster to the largest, this band moves from about 22 to about 21 microns. Except for this gradual shift and the lower intensities for the small clusters, these wavelength spectra are essentially the same for all cluster sizes. The small clusters that were proposed to have
$(\mathrm{MgO})_{\mathrm{n}} \mid \mathrm{R}-\mathrm{REMPI}$

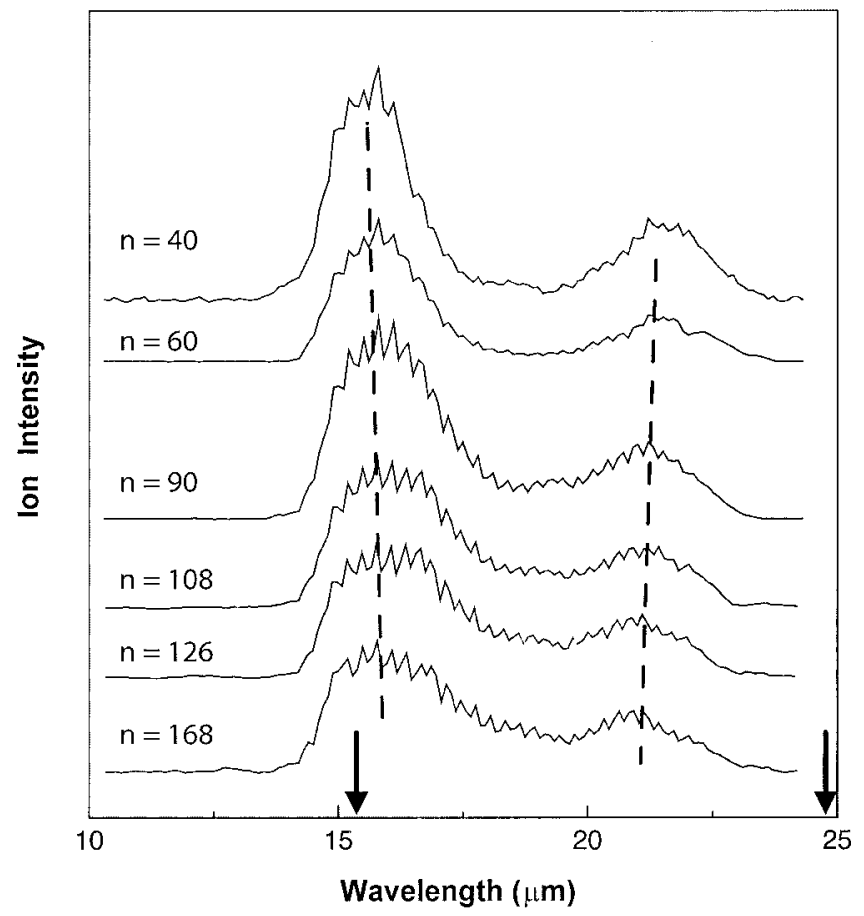

FIG. 4. The wavelength dependence of the IR-REMPI signal for the larger clusters. The arrows indicate the positions of the bulk vibrational modes.

stacked-ring structures have essentially the same spectra as the larger clusters whose masses suggest that they are cubic nanocrystals.

There have been no $a b$ initio calculations to our knowledge that focus on the vibrational spectra of $\mathrm{MgO}$ clusters in either the small or large sizes. To understand these vibrational spectra, therefore, we can only compare to the spectra known for the corresponding bulk material, which has the fcc lattice structure. Bulk and surface phonon vibrational spectra are well characterized for $\mathrm{MgO}$ via infrared reflectance $^{15}$ and high resolution electron energy loss spectroscopy (HREELS) measurements on the solid surface, ${ }^{13,14}$ as well as IR absorption measurements on small particles. ${ }^{18}$ In the HREELS surface phonon spectra, a strong resonance is observed at $651 \mathrm{~cm}^{-1}$ that is assigned as the optically allowed motion of oxygen atoms stretching perpendicular to the surface. Vibrations of bulk material can also be seen in IR reflectance spectroscopy or in the absorption spectra of small particles. However, these latter two measurements are influenced by the combined effects of absorption and reflectance, which depend in a complex way on the surface or particle morphology. Consequently, sharp resonances are not observed in these measurements, but the broad undulating pattern observed must be fit to parallel and perpendicular phonon modes. The perpendicular mode derived is at 640 $\mathrm{cm}^{-1}$, which is a close match with the sharp surface phonon measured by HREELS. These vibrational frequencies match closely with the shorter wavelength feature seen here centered at about 16 microns $\left(625 \mathrm{~cm}^{-1}\right)$. Because these clusters are so small, it is most relevant to compare them to the $\mathrm{MgO}$ surface mode. Compared to this, the IR-REMPI band is 
slightly red-shifted, although the band is quite broad. A redshifted band is in fact expected for the IR-REMPI process due to anharmonicity effects, ${ }^{8(\mathrm{c})}$ and this has been seen in previous spectra of other cluster species. Therefore, the 16 micron band can be assigned as the cluster counterpart of the optically allowed surface phonon that is seen in the HREELS spectrum.

The 22 micron feature is also broad, and it is not as prominent in the smaller clusters. Unlike the 16 micron band, it shifts gradually to the blue with increasing cluster size. The perpendicular phonon for the solid surface is not optically allowed, and therefore it is not seen in the HREELS spectrum. Its frequency is determined to be $401 \mathrm{~cm}^{-1}$ (25 microns) in the small particle absorption data. ${ }^{18}$ It is tempting to associate the 22 micron band seen in the IR-REMPI spectra with this bulk vibration, but the match is not particularly compelling. The frequencies do not match well, and the 22 micron band is shifting further away from the bulk value toward higher frequency as cluster size increases. It is conceivable that the 22 micron band represents a cluster mode that becomes inactive in larger sized particles. On the other hand, it should be noted that the 25 micron bulk phonon band is never observed as a sharp, well defined resonance. It is derived via curve fitting procedures that may have inherent errors associated with them. Considering this, the 22 versus 25 micron difference may not be significant, and it may be that this mode also represents the cluster analogue of this bulk phonon.

If we accept these assignments, then the clusters here are found to have vibrational resonances similar to those in the corresponding $\mathrm{MgO}$ bulk material, which has the fcc crystal structure. This is particularly convincing for the 16 micron feature that is seen as a strong resonance for all clusters studied here and which corresponds to the optically allowed surface phonon seen on the (100) surface. It is therefore safe to assume that these small clusters exhibit this same fcc structural pattern. This is also consistent with the prominent mass peaks observed in the mass distribution, which at higher sizes also suggest cubic structures. On the other hand, small clusters $(n=12,15,18$, etc.) have been suggested in the past to have the nanotube structure composed of stacked six-membered $(\mathrm{MgO})_{3}$ rings. Although these same cluster stoichiometries are measured here, their IR spectra match those of the larger clusters, which also match (except for the 22 versus 25 micron issue) those of the bulk. The simplest conclusion from this is that all $\mathrm{MgO}$ clusters have cubic nanocrystal structures and not nanotube structures. Indeed, the only experimental evidence for the nanotube structure was the numerology, in which multiples of three $\mathrm{MgO}$ units were seen. However, as shown in Table I, all the $n=12,15$, $18,21,24,30$ species can be equally well rationalized with symmetric cubic structures. On the other hand, any conclusion about structure may be premature because we do not know what vibrational spectrum to expect for nanotube clusters. Their vibrations would also involve $\mathrm{Mg}-\mathrm{O}$ stretching motions, and their spectra might not be so different from those seen here, especially considering the width of the bands measured. It is therefore important that $a b$ initio cal- culations investigate the vibrational spectra of both the proposed nanotube and nanocrystal species.

Magnesium oxide in the bulk is well known to form Schottky-type color centers in which oxygen atom vacancies are occupied by electrons. It is interesting to consider whether such defects would occur in clusters, since they occur only rarely in the bulk. While most of the prominent masses seen here can be rationalized as symmetric fcc nanocrystals, there are many weaker intensity peaks that must necessarily correspond to clusters with incomplete or defect nanocrystal structures or which have some other structural growth pattern. To investigate the possibility of other structures perhaps including color centers, we have measured the IR-REMPI spectra for clusters with dimensions close to, but not exactly corresponding to an ideal cubic species. Throughout these data, there is no apparent difference in the resonances observed for these species compared to those already shown in Fig. 4. They all have the same resonances near 16 and 22 microns. In another measurement not shown, we can record the wavelength dependence of the IR-REMPI signal simultaneously for all mass channels to see if any cluster has resonances different from the 16 and 22 micron bands. No other resonances are seen. This implies that all clusters seen here have similar structures, i.e., completed or partially completed fcc nanocrystals. It is somewhat disappointing that we are not able to see any difference between clusters with completed cubic structures and those that must represent growing intermediates. However, this is most likely because the IR active modes with strong intensity are those that have the greatest amplitude motion, i.e., those at the cluster surface. This would be consistent with the optical spectrum of the solid surface. If this is true, then the vibrational frequencies of such surface atoms would not be expected to be very sensitive to structural variations in other parts of the cluster. This trend would increase with cluster size as more surface atoms contribute to the signal. On the other hand, it is also conceivable that the lines observed here are inhomogeneously broadened due to slightly shifted resonances for clusters with the same number of atoms and slightly different structures. To obtain insight on these issues, $a b$ initio calculations are needed that investigate the IR spectra for stacked-ring clusters and for nanocrystal clusters with and without defects.

\section{CONCLUSIONS}

Magnesium oxide clusters containing up to over 300 atoms are produced by laser ablation and studied with ultraviolet ionization and infrared resonance enhanced ionization spectroscopy. Cluster mass spectra in the small size range exhibit somewhat enhanced abundances for stoichiometries corresponding to $(\mathrm{MgO})_{3 n}$ species that have been seen previously, while in the larger size range prominent masses are consistent with cubic nanocrystal structures. These observations are consistent with the results from earlier mass spectrometry studies. IR-REMPI vibrational spectra are obtained for clusters in the size range from about 30 to over 300 atoms. Although previous studies suggested stacked-ring nanotube structures for the small clusters and nanocrystal structures for larger clusters, we find that all clusters have 
essentially the same IR spectra. Two broad resonances near 16 and 22 microns are measured. The 16 micron band occurs at the same position as the optically active perpendicular surface phonon band, while the 22 micron feature is somewhat close to the bulk parallel phonon. Clusters with stoichiometries corresponding to completed cubic structures with roughly equal $x, y, z$ dimensions have essentially the same spectra as those that contain defects or incomplete lattice fragments. However, the spectra are broad and it may be that the linewidth reflects the presence of isomeric structures. $A b$ initio calculations are needed to explore the IR spectra as a function of cluster size and structure.

\section{ACKNOWLEDGMENTS}

The authors gratefully acknowledge the support by the "Stichting voor Fundamenteel Onderzoek der Materie" (FOM) in providing the required beam time of FELIX and greatly appreciate the skillful assistance of the FELIX staff. This work is part of the research program of the FOM, which is supported financially by the "Nederlandse Organisatie voor Wetenschappelijk Onderzoek (NWO)." M.A.D. acknowledges support from the U.S. Department of Energy through Contract No. DE-FG02-96ER14658.

${ }^{1}$ M. M. Kappes, M. Schar, P. Radi, and E. Schumacher, J. Chem. Phys. 84, 1863 (1986); M. M. Kappes, Chem. Rev. 88, 369 (1988).

${ }^{2}$ M. Seidl, K. H. Meiwes-Broer, and M. Brack, J. Chem. Phys. 95, 1295 (1991).

${ }^{3}$ O. Cheshnovsky, K. J. Taylor, J. Conceicao, and R. E. Smalley, Phys. Rev. Lett. 64, 1785 (1990).

${ }^{4}$ H. Wu, S. R. Desai, and L. S. Wang, Phys. Rev. Lett. 77, 2436 (1996); L. S. Wang and H. Wu, Adv. Met. Semicond. Clusters 4, 299 (1998).

${ }^{5}$ R. Busani, M. Folkers, and O. Cheshnovsky, Phys. Rev. Lett. 81, 3836 (1998).

${ }^{6}$ W. A. de Heer, K. Selby, V. Kresin, J. Masui, M. Vollmer, A. Chatelain, and W. D. Knight, Phys. Rev. Lett. 59, 1805 (1987).

${ }^{7}$ J. Tiggesbaumker, L. Koller, H. O. Lutz, and K. H. Meiwes-Broer, Chem. Phys. Lett. 190, 42 (1992).

${ }^{8}$ (a) G. von Helden, I. Holleman, G. M. H. Knippels, A. F. G. van der Meer, and G. Meijer, Phys. Rev. Lett. 79, 5234 (1997); (b) G. von Helden, I. Holleman, M. Putter, A. J. A. van Roij, and G. Meijer, Chem. Phys. Lett. 299, 171 (1999); (c) G. von Helden, I. Holleman, G. Meijer, and B. Sartakov, Opt. Express 4, 46 (1999).

${ }^{9}$ D. van Heijnsbergen, G. von Helden, M. Duncan, A. J. A. van Roij, and G. Meijer, Phys. Rev. Lett. 83, 4983 (1999).

${ }^{10}$ G. von Helden, A. G. G. M. Tielens, D. van Heijnsbergen, M. A. Duncan, S. Hony, L. B. F. M. Waters, and G. Meijer, Science 288, 313 (2000).

${ }^{11}$ G. von Helden, A. Kirilyuk, D. van Heijnsbergen, B. Sartakov, M. A. Duncan, and G. Meijer, Chem. Phys. 262, 31 (2000).

${ }^{12}$ G. von Helden, D. van Heijnsbergen, M. A. Duncan, A. J. A. van Roij, and G. Meijer, Chem. Phys. Lett. 333, 350 (2001).

${ }^{13}$ V. E. Henrich and P. A. Cox, The Surface Science of Metal Oxides (Cambridge University Press, Cambridge, MA, 1994).

${ }^{14}$ P. A. Cox and A. A. Williams, J. Electron Spectrosc. Relat. Phenom. 39, 45 (1986).

${ }^{15}$ C. F. Bohren and D. R. Huffman, Absorption and Scattering of Light by Small Particles (Wiley Interscience, New York, 1983).
${ }^{16}$ The Surface Science of Metal Oxides, Faraday Discussion No. 114 (The Royal Society of Chemistry, London, 1999).

${ }^{17}$ G. E. Brown, V. E. Henrich, W. H. Casey et al. Chem. Rev. 99, 77 (1999).

${ }^{18}$ S. Hiyashi, N. Nakamori, J. Hirono, and H. Kanamori, J. Phys. Soc. Jpn. 43, 2006 (1977)

${ }^{19}$ Handbook of Chemistry and Physics, 79th ed., edited by David R. Lide (CRC Press, Baton Rouge, LA, 1999).

${ }^{20}$ H. Y. Afeefy, J. F. Liebman, and S. E. Stein, "Neutral thermochemical data" in NIST Chemistry WebBook, NIST Standard Reference Database Number 69, edited by P. J. Linstrom and W. G. Mallard (National Institute of Standards and Technology, Gaithersburg, MD 2001) (http:// webbook.nist.gov).

${ }^{21}$ K. P. Huber and G. Herzberg, Constants of Diatomic Molecules (Van Nostrand Reinhold, New York, 1979).

${ }^{22}$ D. Bellert, K. L. Burns, R. Wampler, and W. H. Breckenridge, Chem. Phys. Lett. 322, 41 (2000).

${ }^{23}$ W. A. Saunders, Phys. Rev. B 37, 6583 (1988); W. A. Saunders, Z. Phys. D: At., Mol. Clusters 12, 601 (1989).

${ }^{24}$ P. J. Zieman and A. W. Castleman, Jr., J. Chem. Phys. 94, 718 (1991); P. J. Zieman and A. W. Castleman, Jr., Z. Phys. D: At., Mol. Clusters 20, 97 (1991); H. T. Deng, Y. Okada, M. Foltin, and A. W. Castleman, Jr., J. Phys. Chem. 98, 9350 (1994); P. J. Zieman and A. W. Castleman, Jr., Phys. Rev. B 44, 6488 (1991).

${ }^{25}$ M. Gutowski, P. Skurski, X. Li, and L. S. Wang, Phys. Rev. Lett. 85, 3145 (2000).

${ }^{26}$ J. H. Kim, X. Li, L. S. Wang, H. L. de Clercq, C. A. Fancher, O. C. Thomas, and K. H. Bowen, J. Phys. Chem. 105, 5709 (2001).

${ }^{27}$ S. Moukouri and C. Noguera, Z. Phys. D: At., Mol. Clusters 24, 71 (1991)

${ }^{28}$ A. I. Boldyrev, I. L. Shamovsky, and P. v. R. Schleyer, J. Am. Chem. Soc. 114, 6469 (1992).

${ }^{29}$ A. I. Boldyrev, J. Simons, and P. v. R. Schleyer, Chem. Phys. Lett. 233, 266 (1995).

${ }^{30}$ J. M. Recio, R. Pandey, A. Ayuela, and A. B. Kunz, J. Chem. Phys. 98, 4783 (1993).

${ }^{31}$ E. D. L. Puente, A. Aguado, A. Ayuela, and J. M. Lopez, Phys. Rev. B 56, 7607 (1997); A. Aguado and J. M. Lopez, J. Phys. Chem. B 104, 8398 (2000).

${ }^{32}$ M. J. Malliavan and C. Coudray, J. Chem. Phys. 106, 2323 (1997).

${ }^{33}$ M. Wilson, J. Phys. Chem. B 101, 4917 (1997).

${ }^{34}$ T. Henning, Chem. Soc. Rev. 27, 315 (1998).

${ }^{35}$ A. G. G. M. Tielens, L. B. F. M. Waters, F. J. Molster, and K. Justtanont, Astrophys. Space Sci. 255, 415 (1998).

${ }^{36}$ T. Posch, F. Kerschbaum, H. Mutschke, D. Fabian, J. Dorschner, and J. Hron, Astron. Astrophys. 352, 609 (1999).

${ }^{37}$ E. F. van Dishoeck and A. G. G. M. Tielens, in The Century of Space Science, edited by J. Bleeker, J. Geiss, and M. C. E. Huber (Kluwer, Dordrecht, in press).

${ }^{38}$ A. Amrein, R. Simpson, and P. Hackett, J. Chem. Phys. 95, 1781 (1991).

${ }^{39}$ T. Leisner, K. Athanassenas, D. Kreisle, E. Recknagel, and O. Echt, J. Chem. Phys. 99, 9670 (1993).

${ }^{40}$ E. E. B. Campbell, G. Ulmer, and I. V. Hertel, Phys. Rev. Lett. 67, 1986 (1991).

${ }^{41}$ P. Wurz and K. R. Lykke, J. Chem. Phys. 95, 7008 (1991).

${ }^{42}$ B. D. May, S. F. Cartier, and A. W. Castleman, Jr., Chem. Phys. Lett. 242, 265 (1995).

${ }^{43}$ S. F. Cartier, B. D. May, and A. W. Castleman, Jr., J. Chem. Phys. 104, 3423 (1995)

${ }^{44}$ D. Oepts, A. F. G. van der Meer, and P. W. van Amersfoort, Infrared Phys. Technol. 36, 297 (1995).

${ }^{45}$ K. F. Willey, K. LaiHing, T. G. Taylor, and M. A. Duncan, J. Phys. Chem. 97, 7435 (1993) 